\title{
ON THE FORMATION OF CARBON STARS
}

\author{
Greg L. Bryan and KeVIN VolK ${ }^{1}$ \\ Department of Physics and Astronomy, The University of Calgary \\ AND \\ SUN KWOK ${ }^{2}$ \\ Joint Institute for Laboratory Astrophysics, National Institute of Standards and Technology and University of Colorado \\ Received 1990 February 20; accepted 1990 May 21
}

\begin{abstract}
We present an integrated model of asymptotic giant branch (AGB) evolution, using empirical formulae to allow for nuclear shell burning in the core, the dredge-up of heavy elements to the envelope, and mass loss from the surface. We emphasize the role of mass loss in the formation of carbon stars. The formation rate of carbon stars, for an assumed constant dredge-up every thermal pulse, is critically dependent on the mass-loss formula. The observed luminosity distribution of AGB stars, the initial-final mass relationship, the oxygenrich-to-carbon-rich star ratio, and the total population of carbon stars can be reproduced by a new proposed mass-loss formula based upon the Reimers formula but with an initial mass- $\left[M_{*}(0)\right]$ dependent $\eta$ value $(\eta=$ $\left.0.2875\left[M_{*}(0)^{2}-10.6 M_{*}(0)+10.2\right]\right)$. For initial masses between 1.25 and $8 M_{\odot}$, our best fit is obtained with a carbon dredge-up of $\sim 6 \%$ by mass each thermal pulse. The observed data are, however, not consistent with any of the "superwind" mass-loss formulae where most of the envelope mass is removed near the end of the AGB. We also find that carbon stars primarily descended from a low-mass $\left(<3 M_{\odot}\right)$ population, and highmass (3-8 $\left.M_{\odot}\right)$ stars remain oxygen-rich for most of their AGB lifetime.
\end{abstract}

Subject headings: stars: carbon - stars: circumstellar shells - stars: evolution - stars: mass loss

\section{INTRODUCTION}

Stars on the asymptotic giant branch (AGB) can be readily classified as either oxygen-rich (M) or carbon-rich (C) based on the abundance ratio of carbon and oxygen atoms. Atoms of the less abundant element are tied up in $\mathrm{CO}$ molecules, and the excess atoms of the more abundant element form molecules which have distinct absorption bands in the visible part of the spectrum. Spectra of $\mathbf{M}$ stars are dominated by metal oxide bands such as TiO, ZO, and YO whereas $\mathrm{C}$ stars have bands of $\mathrm{C}_{2}$ and $\mathrm{CN}$. It is believed that $\mathrm{C}$ stars are created when significant numbers of carbon atoms are dredged up from the core by the deep convective envelope developed after a helium shell flash, which is also referred to as a thermal pulse. The steady increase of the abundance of carbon in the photosphere eventually converts an $\mathbf{M}$ star to a $\mathbf{C}$ star.

Quantitative models of the dredge-up theory, however, predict that carbon stars can only form at high luminosities (Iben 1981; Renzini and Voli 1981), but the actual observed luminosities of $\mathrm{C}$ stars are much lower (Blanco, McCarthy, and Blanco 1978, 1980; see Iben 1988 and references therein). There are a number of uncertain physical processes that could affect the theoretical results. These include carbon recombination (Iben and Renzini 1982), the mixing-length parameter (Boothroyd and Sackmann 1988c), semiconvection (Hollowell 1987), convective overshooting (Paczyński 1977; Chiosi, Bertelli, and Bressan 1987), core breathing pulses (Castellani et al. 1985), etc. It is encouraging to note that some progress has been made recently (Lattanzio 1989a), but the complexity of the problem makes it unlikely that any definitive conclusion can be made on the dredge-up theory in the near future.

\footnotetext{
${ }^{1}$ Canadian Institute for Theoretical Astrophysics National Fellow, 19891991.

${ }^{2}$ JILA Visiting Fellow 1989-1990, on leave from University of Calgary, Calgary, Alberta, Canada.
}

If we leave the details of the dredge-up process aside and consider the formation of carbon stars, there is another physical process that has a major effect on when carbon stars are formed on the AGB. We now know that all $M$ stars with spectral types later than M3 suffer from large-scale mass loss from the surface. Mass loss has the effect of (1) reducing the mass of the hydrogen envelope and therefore making dredge up more difficult (Wood 1981), (2) reducing the number of thermal pulses needed to make the photosphere carbon rich, and (3) terminating the AGB at lower luminosities (Lattanzio $1989 b$ ). On the observational side, mass loss also creates thick circumstellar envelopes that make the discovery of evolved $M$ and $C$ stars difficult, at least in external galaxies where few infrared data are available. In this paper, we will explore the effects of mass loss on the formation of carbon stars in solar metallicity stars. In order to isolate the effects of mass loss and to avoid the uncertainties in the dredge-up theory, we will simply assume that the dredge-up rate is constant for every thermal pulse.

While past studies of the $\mathrm{C}$ stars problem have relied mostly on data in the visible part of the spectrum, recent studies of the circumstellar properties of AGB stars using data from the Infrared Astronomical Satellite (IRAS) have established two important facts. (1) There exist large numbers of evolved $M$ stars losing mass at a very high rate as evidenced by the deep absorption feature of silicate dust in the circumstellar envelope. It is likely that these stars are near the end of the AGB and will evolve directly to planetary nebulae without ever entering the C star phase. (2) There are two kinds of carbon stars, one identified by their photospheric spectra (hereafter visual $\mathrm{C}$ stars) and the other identified by their infrared circumstellar features (hereafter infrared $\mathrm{C}$ stars). Infrared $\mathrm{C}$ stars have very red infrared colors and are undergoing mass loss at a very high rate, whereas visual $\mathrm{C}$ stars generally have far infrared excesses and are not currently losing mass (Thronson et al. 1987; Chan 
and Kwok 1988). The far-infrared excesses observed in visual C stars suggest that these stars descend from mass-losing $M$ stars (Willems and de Jong 1988). There also exists a continuous evolution sequence from visual $C$ stars to infrared $C$ stars (Chan and Kwok 1990). The large mass-loss rates observed in infrared $\mathrm{C}$ stars suggest that they will soon deplete the entire hydrogen envelope and leave the AGB.

The above observational facts point to an inescapable conclusion: some AGB stars stay oxygen-rich, while some $M$ stars evolve into $C$ stars and terminate the AGB as C stars (Kwok, Volk, and Chan 1989). This implies a branching on the AGB, but which parameters determine the branching is unclear. It is likely that one of the major parameters will be the initial mass. Stars with high initial masses will have larger hydrogen envelopes and therefore will take more thermal pulses to become carbon-rich. At the same time, the high luminosities of these stars cause high mass-loss rates and greatly shorten their AGB lifetime. In this case, only stars with initial masses lower than a certain limit will become $\mathrm{C}$ stars. It is also possible that there exists a lower mass limit below which there is insufficient dredge-up as in some current theories (Renzini and Voli 1981).

In order to quantitatively evaluate the effects of mass loss on the formation of $\mathrm{C}$ stars, it is necessary to have a knowledge of the mass-loss formula on the AGB. In recent years, a number of mass-loss formulae have been proposed. These formulae vary greatly but can be categorized by whether they assume that mass loss is continuous over the entire AGB or has a sudden discontinuity near the end of the AGB. In this paper, we will use different mass-loss formulae and investigate their effects on $\mathrm{C}$ star formation. The results will then be compared to the observed properties of $\mathrm{C}$ stars.

\section{MASS LOSS ON THE ASYMPTOTIC GIANT BRANCH}

As AGB stars are often obscured by thick circumstellar dust envelopes, their evolution can best be traced by their infrared properties. Oxygen-rich stars are characterized by the presence of the silicate feature, either in emission or in absorption (Merrill 1977). As the stars ascend the AGB, the mass-loss rate increases leading to larger optical depths in the circumstellar envelope. The silicate feature will change from emission to absorption and the continuum color will change from 600 $1000 \mathrm{~K}$ to about $300-600 \mathrm{~K}$. A first effort to model this sequence was made by Bedijn (1987). He assumed that mass loss on the AGB follows the Reimers formula with $\eta=1$ until $\dot{M}=10^{-6} M_{\odot} \mathrm{yr}^{-1}\left(=\dot{M}_{0}\right)$, after which time the mass-loss formula goes as

$$
\dot{M}_{e}=-\dot{M}_{0}(1-t / \tau)^{-1},
$$

where $M_{e}$ is the envelope mass and $\tau$ is a time constant set at $\sim 10^{5} \mathrm{yr}$ depending on the initial mass. With this formula, Bedijn (1987, hereafter B87) was able to fit the energy distribution of $\mathrm{OH} / \mathrm{IR}$ stars.

A similar approach was taken by Volk and Kwok (1988, hereafter VK) who assumed a mass loss formula

$$
\dot{M}=-1.8 \times 10^{-12}\left[M_{*}(0) / 8\right] L_{*} R_{*} M_{*}^{-1} M_{\odot} \mathrm{yr}^{-1}
$$

throughout the AGB. In this formula, $L_{*}, R_{*}$, and $M_{*}$ are, respectively, the luminosity, radius, and total mass of the star in solar units, and $M_{*}(0)$ is the initial mass (in solar units) of the star before mass loss. During the AGB, $L_{*}$ (and therefore $R_{*}$ ) increases as the result of nuclear burning and $M_{*}$ decreases as the result of mass loss. The evolution of the circumstellar spectra is therefore coupled with the evolution of the star. The model spectra are fitted to the AGB stars with IRAS lowresolution spectra as well as convolved with $I R A S$ instrumental profiles to simulate the observed color distributions of AGB stars. In the models of both B87 and VK, model evolutionary tracks can be calculated which follow the change in continuum colors of oxygen-rich AGB stars.

The case for $\mathrm{C}$ stars is more complicated, however. Visual carbon stars are easily identified by objective prism surveys and thousands of Galactic visual $\mathrm{C}$ stars have been catalogued (Stephenson 1973; MacConnell 1988). Because of the heavy circumstellar extinction, infrared $\mathrm{C}$ stars are mostly discovered as the result of infrared sky surveys such as the Two Micron, AFGL, and IRAS sky surveys. As the result of the IRAS survey, several hundred infrared carbon stars are now known (Little-Marenin 1986; Walker and Cohen 1988). It has been recently suggested that visual carbon stars represent a period where mass loss is interupted, following a mass-losing episode as an M siar (Willems and de Jong 1988; Chan and Kwok 1988). The visual $\mathrm{C}$ star stage is followed by another episode of mass loss, this time with carbon-based grains. The dust envelope formed by this second mass-loss episode will show the $\mathrm{SiC}$ feature and the stars be observed as infrared $\mathrm{C}$ stars. The mass-loss process will continue until the entire hydrogen envelope has been depleted by mass loss.

While the observed colors of $\mathbf{M}$ and $\mathrm{C}$ stars can be successfully modeled by the above mass-loss scenarios (Kwok, Volk, and Chan 1989), there is no integrated evolutionary picture that ties the $M$ and $C$ stars together. In this paper, we assume that mass loss starts at the beginning of the thermalpulsing AGB. Assuming a simple dredge-up formula, the envelope will be increasingly enriched in successive thermal pulses. When the carbon abundance in the envelope exceeds that of oxygen, the star will become a $\mathrm{C}$ star. It is also assumed at this point that mass loss ceases, probably because all oxygen atoms are tied up in $\mathrm{CO}$ and are not available for the formation of silicate grains which are necessary to drive mass loss. After an interval of $10,000 \mathrm{yr}$, mass loss resumes again. The mass-loss rates during both the $M$ and $C$ star phases are assumed to be governed by the same formula which is dependent on the stellar parameters $L_{*}, R_{*}, M_{*}$, etc. A number of dredge-up and mass-loss formulae will be explored and the resultant formation rate of $\mathrm{C}$ stars discussed.

\section{MODELS}

A complete theory of AGB evolution requires the integration of the nuclear shell burning in the core, dredge-up of heavy elements in the convective envelope, mass loss from the surface, and radiative transfer effects in the circumstellar envelope. To treat every aspect of the above in detail would be an impossible task. In this paper, we have extracted results from existing models and attempted to present an overall picture which is consistent with our present observational knowledge of AGB stars based on their photospheric and circumstellar properties. Details of the mathematical formulations are given below.

\section{a) Evolution on the $A G B$}

The beginning of the AGB can be identified with the end of core helium burning. The evolutionary stage that comes after is referred to as the early AGB (Iben and Renzini 1983). We will define the first helium flash (thermal pulse) as our zero point in 
time $(t=0)$, and mass loss is assumed to begin at this point. The initial masses of the core $\left[M_{c}(0)\right]$ have been calculated for low-mass $\left(1-3 M_{\odot}\right)$ stars of solar abundances by Boothroyd and Sackmann (1988b). The corresponding values for higher mass stars (3-9 $M_{\odot}$ ) are given by Iben and Renzini (1983). In order to join the two regimes together, we have adopted the following interpolations:

$$
\begin{array}{ll}
M_{c}(0)=0.483+0.021 M_{*}(0), & 1.25<M_{*}(0)<3 \\
M_{c}(0)=0.546+0.087\left\{\left[M_{*}(0) / 3\right]^{2}-1\right\} & 3<M_{*}(0)<9 .
\end{array}
$$

During the interpulse period, the stellar luminosity can be approximated by a core mass-luminosity relationship (Paczyński 1971). The following empirical formulae are adopted based on the core mass-luminosity relationships given in Boothroyd and Sackmann (988a):

$$
\begin{array}{ll}
L_{*}=38,480\left(M_{c}-0.434\right), & 0.434<M_{c}<0.519 \\
L_{*}=52,000\left(M_{c}-0.456\right), & 0.519<M_{c}<0.775 \\
L_{*}=59,250\left(M_{c}-0.495\right), & 0.775<M_{c}<0.9 \\
L_{*}=65,000\left(M_{c}-0.543\right), & 0.9<M_{c}<1.41 .
\end{array}
$$

Since mass of the core increases as the result of nuclear burning, the rate of change in the mass of the core can be expressed as

$$
\dot{M}_{c}=\left(L_{*} / x E_{\mathrm{H}}\right)=a\left(M_{c}-b\right)
$$

where $x(=0.7)$ is the hydrogen mass abundance, $E_{\mathrm{H}}=6$ $\times 10^{18}$ ergs is the energy released by converting $1 \mathrm{~g}$ of hydrogen to helium, and $a$ and $b$ are constants. Substituting equation (4) into equation (5), equation (5) can be integrated to obtain the change of core mass with time. Assuming a certain massloss formula, we see that the mass of the envelope $\left(M_{e}\right)$ is given by

$$
M_{e}(t)=M_{e}(0)+\int \dot{M}_{e}(t) d t .
$$

The total mass of the star is then given by $M_{*}=M_{c}+M_{e}$. The other stellar parameters can be derived accordingly:

$$
\log _{10}\left(T_{*}\right)=\left[101.49-2.5 \log _{10}\left(L_{*}\right)+0.533 M_{*}\right] / 26.64
$$

(Wood and Cahn 1977)

$$
R_{*}(t)=\left\{L_{*}(t) /\left[4 \pi \sigma T_{*}^{4}(t)\right]\right\}^{1 / 2},
$$

where $T_{*}$ is the effective temperature of the star, and $\sigma$ is the Stephan-Boltzmann constant.

\section{b) Mass-Loss Formulae}

We assume that mass loss begins at $t=0$, the same time as thermal pulses begin. For low-mass stars, the horizontalbranch morphology suggests that $\sim 0.2 M_{\odot}$ is lost on the red giant branch (Iben and Rood 1970). In this paper, red giant branch mass loss is taken into account by the following formula:

$$
\begin{aligned}
\Delta M_{e} & =0.483 M_{*}(0)-0.161 M_{*}(0)^{2} & & M_{*}(0)<3 \\
& =0, & & M_{*}(0)>3 .
\end{aligned}
$$

For mass loss on the AGB, a number of different mass-loss formulae will be considered. In addition to the mass-loss formula of B87 and VK, we have also included the mass-loss formula of Baud and Habing (1983, hereafter BH)

$$
\dot{M}_{e}=-4 \times 10^{-13}\left[M_{e}(0) / M_{e}(t)\right] L_{*} R_{*} M_{*}^{-1} M_{\odot} \mathrm{yr}^{-1} \text {. }
$$

and a modified version of the mass-loss formula of VK. The different behaviors of the mass-loss formulae are given in Figure 1. The formulae from $\mathrm{BH}$ and $\mathrm{B} 87$ are similar in that the mass-loss rate goes up to a very high value over a short period of time near the end of the AGB. This is sometimes referred to as the "superwind phase."

One possible observational test of the mass-loss formula is through the initial-final mass relationship. Based on the observations of white dwarfs in open clusters, an empirical relationship has been obtained by Weidemann and Koester (1983). For comparison, the theoretical initial-final mass relationships calculated from the mass-loss formulae are plotted in Figure 2. We can see that the theoretical relationships are all approximately linear in form, while the observed relationship is better fitted by a power law of index $\sim 2$. This implies that stars with low initial masses $\left(2-5 M_{\odot}\right)$ probably suffer relatively higher rates of mass loss than assumed in the three mass-loss formulae given above. To take this into consideration, we have modified the formula of $\mathrm{VK}$ into the following form by adding a quadratic term in $M_{*}(0)$ :

$$
\begin{array}{rl}
\dot{M}_{e}=-1.15 \times 10^{-13}\left[M_{*}(0)^{2}-\right. & \left.10.6 M_{*}(0)+10.2\right] \\
& \times L_{*} R_{*} M_{*}^{-1} M_{\odot} \mathrm{yr}^{-1}, \\
1 & 1.25 M_{\odot}<M_{*}<8 M_{\odot} .
\end{array}
$$

The lower mass limit of $1.25 M_{\odot}$ corresponds to the mainsequence turnoff mass of the present epoch. Figure 2 shows that the initial mass-final mass relationship predicted by equation (11) is closer to the observed relationship of Weidemann and Koester (1983).

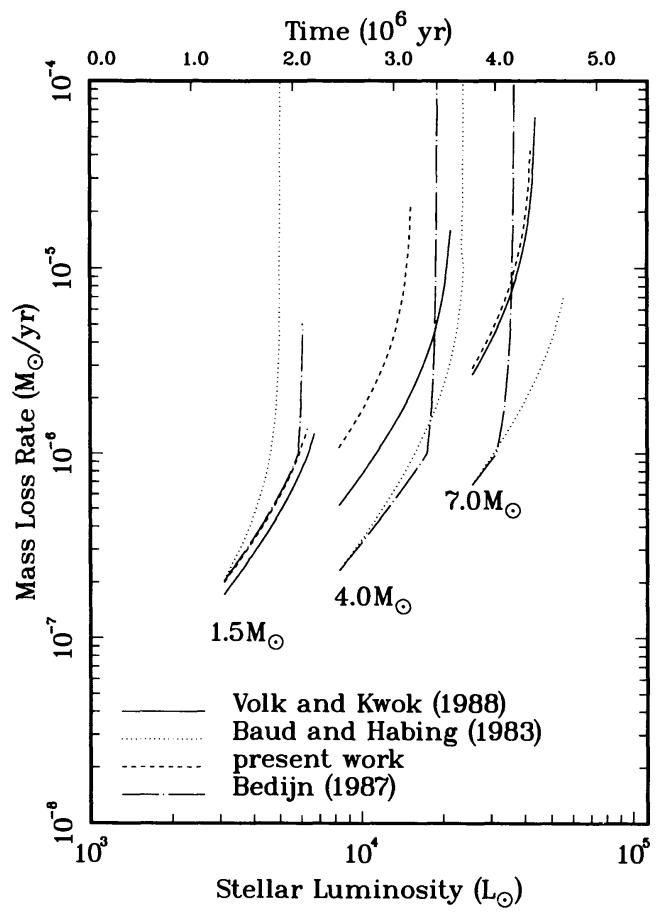

Fig. 1.-The four mass-loss formulae used in this paper for initial stellar masses of $1.25,4$, and $7 M_{\odot}$. 


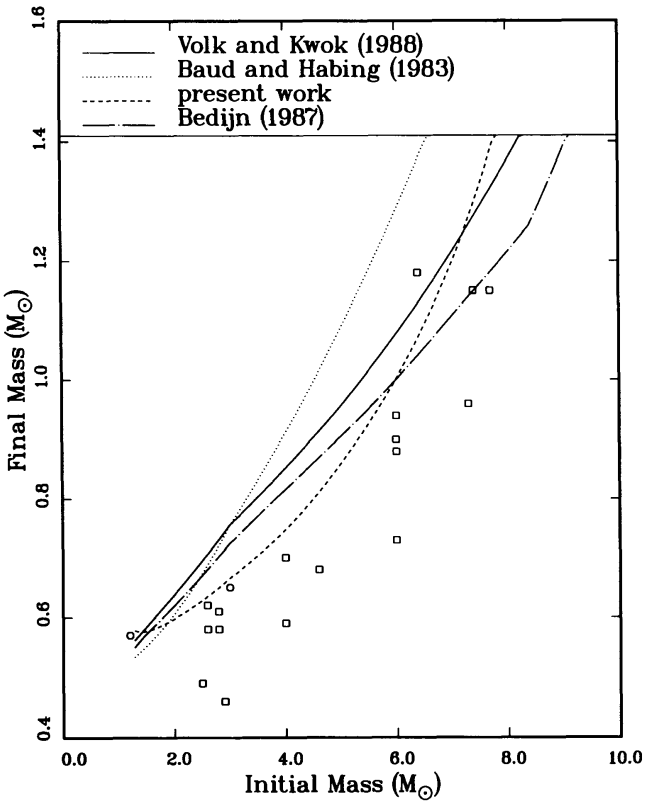

Fig. 2.-Initial-final mass relationships of the four mass loss formulae. The squares are observational data taken from Weidemann and Koester (1983), and the circles are from Boothroyd and Sackmann (1988b).

\section{c) Dredge-up Model}

The formation of carbon stars is assumed to be due to the dredge-up of carbon (as well as other s-process elements) that occurs when the convective envelope in the outer hydrogen shell reaches into the region between the $\mathrm{C}-\mathrm{O}$ core and hydrogen shell (Iben 1982). This takes place immediately following a thermal pulse. There is much debate concerning the details of this process, especially in regard to how much material is brought up and on which pulses it occurs (Boothroyd and Sackmann 1988c; Lattanzio 1989a). For simplicity, we assume that dredge up occurs on every pulse and a constant fraction $(\chi)$ of the interpulse region is brought up as carbon. The amount of carbon dredge up after each thermal pulse can be written as

$$
M_{\text {carbon }}=\chi \int \dot{M}_{c} d t,
$$

where the integral is over the interpulse period. The existence of a relationship between $\Delta t$ and $M_{c}$ was first noted by Paczyński (1975). In this paper, we have adopted the formula of Boothroyd and Sackmann (1988b) for stars of solar abundance. During the thermal pulse, a fraction of the carbon produced by helium burning is dredged up to the envelope. In the envelope, mass loss is assumed to deplete all elements equally. Since $M_{\text {carbon }}$ of carbon is added after each pulse, the abun-
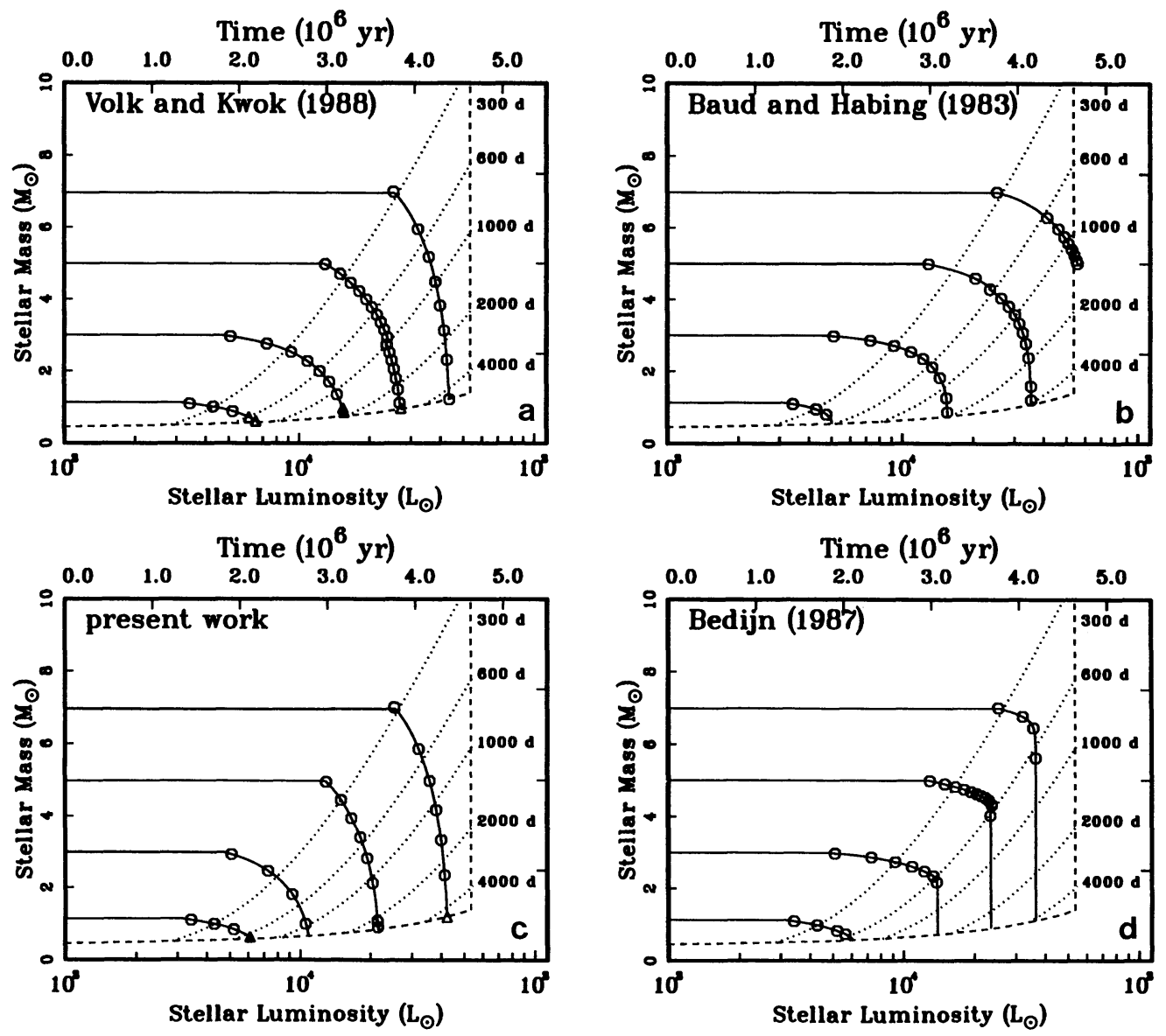

FIG. 3. Change in the total mass as a function of time for stars of initial masses $1.5,3,5$, and $7 M_{\odot}$. Diagrams $(a)-(d)$ correspond to the four mass-loss formulae. The value of $\chi$ assumed to be 0.06 . Also plotted in the diagrams are the evolution of the core mass (curved dashed line), the Chandrasekhar limit (vertical dashed line), and curves of constant $(300,600,1000,2000$, and 4000 days) pulsation periods in fundamental mode (dotted lines). 
dance of carbon in the envelope will eventually exceed that of oxygen, therefore creating a $\mathrm{C}$ star.

The effects of mass loss on the formation of $\mathrm{C}$ stars can be graphically illustrated in a mass-luminosity diagram first used by Wood and Cahn (1977). Figure 3 shows the change in stellar mass as the star ascends the AGB. The evolutionary tracks for four initial masses for each of the four mass-loss formulae are shown. When the thermal pulse occurs during the oxygen-rich phase, the curve is marked by a circle beginning with the first pulse. This symbol changes to a triangle when the star becomes carbon-rich. The number of thermal pulses between symbols are $2,5,10$, and 200 for $1.5,3,5$, and $7 M_{\odot}$, respectively. The only exception is for the $\mathrm{BH}$ case, where the corresponding numbers are 2, 5, 50, and 1000 . The last thermal pulse is always plotted to indicate the final state of the star.

We can see that the two "superwind" formulae (B87 and $\mathrm{BH})$ have difficulty producing $\mathrm{C}$ stars. For low-mass stars, mass loss depletes the envelopes too early before dredge up can have an effect; and for high-mass stars, large-scale mass loss develops too late to overcome the large envelope mass.

We have experimented with different values of $\chi$ in the production of C stars. The fraction of a star's AGB lifetime as a C star under different assumed parameters are shown in Figure 4. In all mass-loss formulae, high-mass stars generally stay oxygen-rich throughout their AGB lifetime. This is the result of the large envelope mass diluting the amount of carbon dredged-up. The behavior of $\mathrm{BH}$ formula is qualitatively different from the others in that the $\mathrm{C}$ stars primarily are produced from high-mass progenitors. The formula of B87 can produce C stars, but only at high $(\sim 0.1) \chi$ values.

\section{d) Luminosity Function of Carbon Stars}

To explain the number of $\mathrm{C}$ stars in the Galaxy, one has to take into account the mass distribution of the initial masses of AGB stars. For this purpose, we have used the initial mass function $\left(d N / d m \propto m^{-2.35}\right)$ of Salpeter (1955). The rate of stars entering the AGB is assumed to be one per year in the Galaxy and the main-sequence turn-off mass is $1.25 M_{\odot}$ (Miller and Scalo 1979). Figure 5 shows the C-to-M star ratio as a function of $\chi$. The observed Galactic $\mathrm{C}$ to $\mathrm{M}$ star ratio of $\sim 0.1$ (Richer and Crabtree 1985) can be reproduced by a dredge-up parameter $\chi$ of $\sim 0.065$ in our model. For the formulae of B87 and $\mathrm{BH}$, the ratio of $\mathrm{C}$ to $\mathrm{M}$ stars is small unless $\chi$ is unrealistically large.

Figures 6 and 7 show respectively the luminosity functions for $\mathrm{M}$ and $\mathrm{C}$ stars for $\chi=0.06$. The luminosity function is derived by convolving the initial mass function with the amount of time stars of each initial mass spend at each luminosity interval. The distribution for $\mathbf{M}$ stars has a "tail" extending to the AGB limit whereas the distribution for $\mathrm{C}$ stars is relatively narrow and peaks at $M_{\text {bol }} \sim-4.7$. While the luminosity functions for $M$ stars are similar for the four mass-loss formulae, the $\mathrm{C}$ star distributions are very different. Significant number of $C$ stars are produced by VK and equation (11), but the luminosity function is narrower in the latter case. The mass-loss formula of $\mathrm{B} 87$ has the correct shape for the lumin-
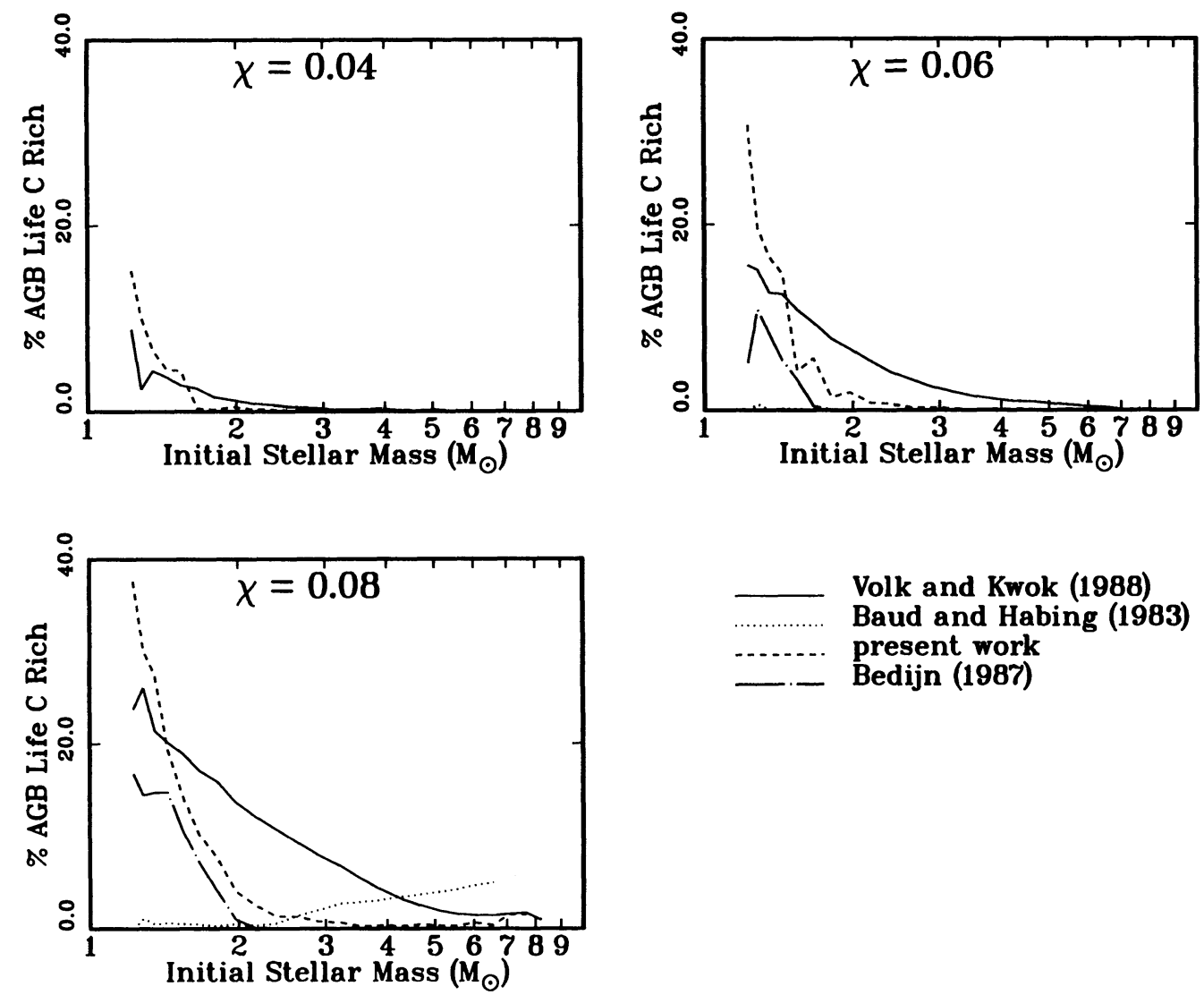

Volk and Kwok (1988)
Baud and Habing (1983)
present work present work
Bedijn (1987)

FIG. 4. - The predicted fraction of AGB lifetime that a star spends as a $C$ star as a function of initial mass for three values of $\chi$ 


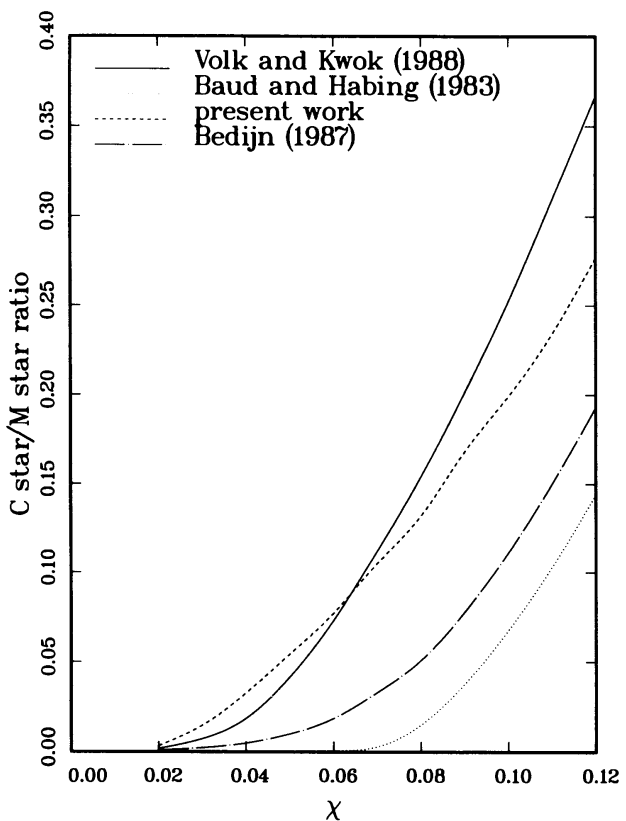
er $\chi$ osity function but the total number of $\mathrm{C}$ stars produced is smaller. The formula of $\mathrm{BH}$ produces hardly any $\mathrm{C}$ stars.

\section{RESULTS AND DISCUSSION}

\section{a) Luminosity Function of Carbon Stars}

Due to distance uncertainties, the luminosity function of Galactic carbon stars is not easy to determine. However, carbon stars are easy to identify in external galaxies (Richer 1981), especially in the Magellanic Clouds. Surveys of carbon stars in the Magellanic Clouds shows a luminosity function peaked at $M_{\text {bol }} \sim-4.8$, and no carbon stars are seen with $M_{\text {bol }}<-6$ (Cohen et al. 1981; Wood, Bessell, and Fox 1983; Wood 1987). This was in complete contradiction of the carbon star theories at the time, which predicted that all carbon stars should have luminosities greater than $M_{\text {bol }}=-6$. In comparison, the present model results as shown in Figure 7 give a much better representation of the observed luminosity function.

When making comparisons between the present models and the observed luminosity functions in the Magellanic Clouds, one has to bear in mind the difference in metallicity between the Clouds and the Galaxy. The mass-loss formulae used here are derived from observations of Galactic AGB stars, which may have higher mass-loss rates than those in the Clouds
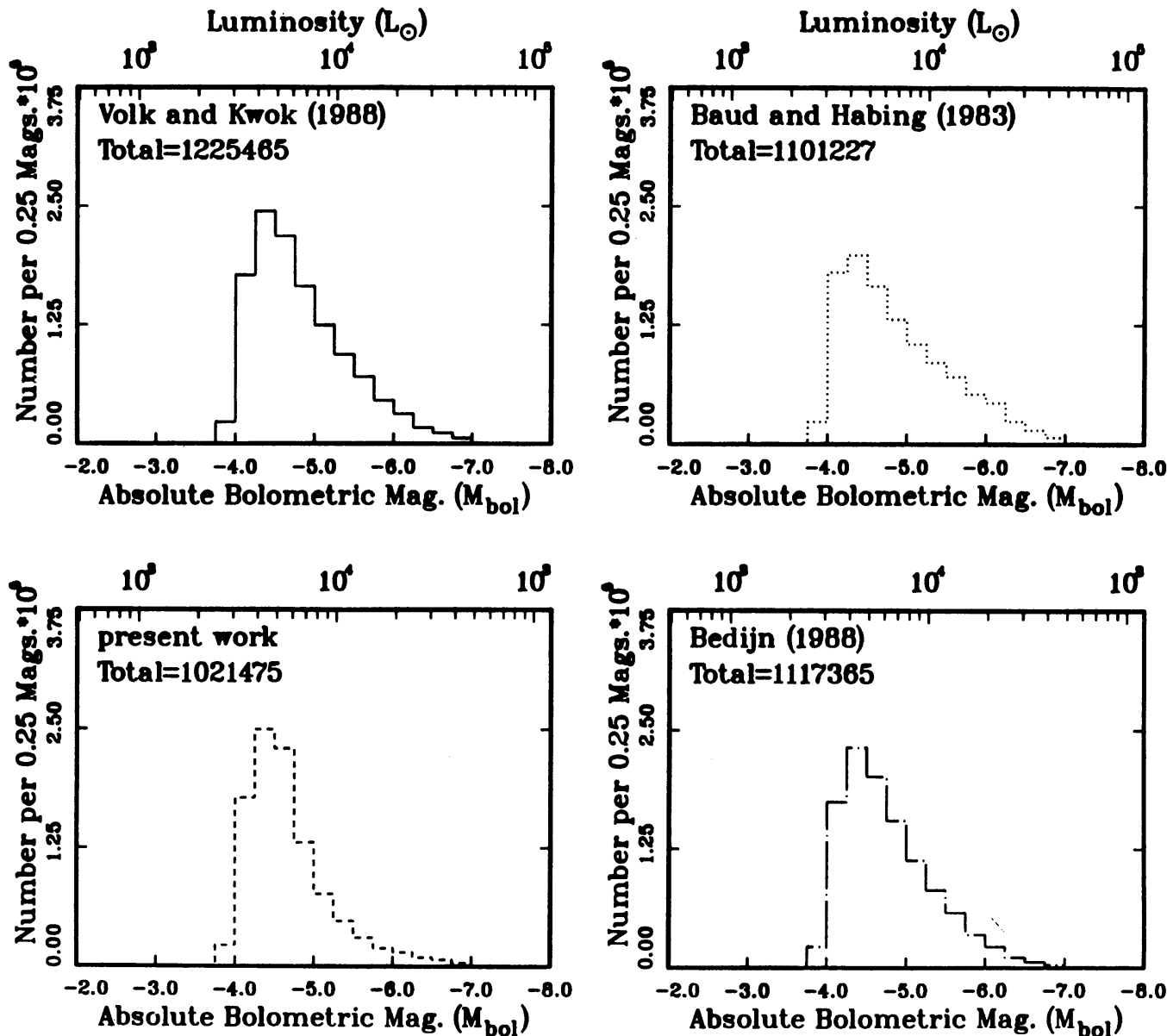

Fig. 6.-Model luminosity functions of $M$ stars. The value of $\chi$ is 0.06 . The luminosities in solar units are plotted on the top scale. The totals shown in the upper right-hand corners are the total number of $M$ stars in the Galaxy assuming a main-sequence turn-off rate of 1 star per year in the Galaxy. 

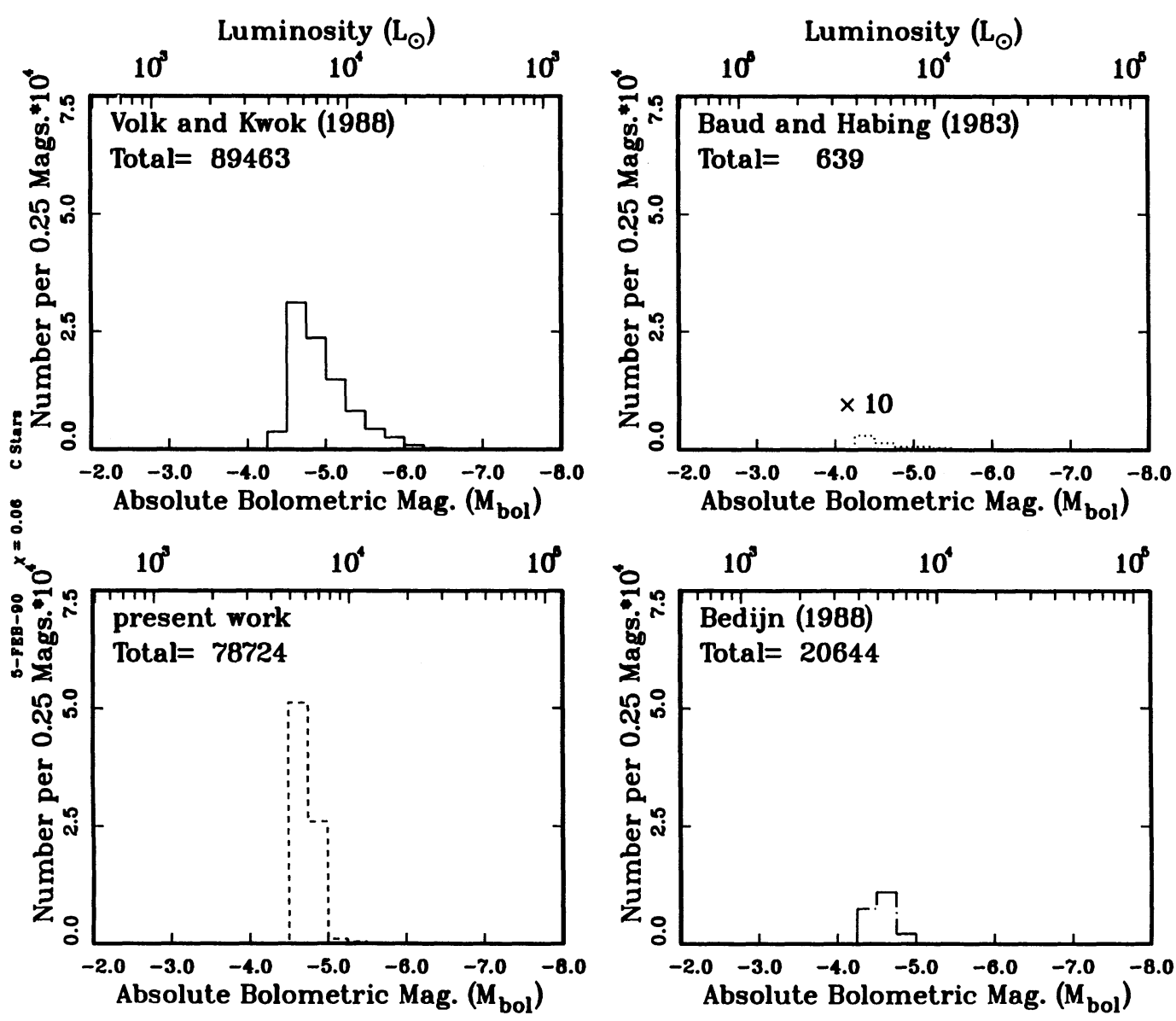

Fig. 7. - Model luminosity function of $\mathrm{C}$ (visual + infrared) stars. Parameters are the same as in Fig. 5.

because of the metallicity difference. If this is the case, then the luminosity function for the Clouds would peak at higher luminosity than for the Galaxy.

It is clear that neglecting or underestimating of the magnitude of mass loss can contribute to the failure of current theories. Mass loss is certainly responsible for the removal of $\mathrm{C}$ stars at high luminosities, either by early termination of the $\mathrm{AGB}$, or by converting them to infrared $\mathrm{C}$ stars which would be difficult to detect in external galaxies. With the inclusion of mass loss, it is now possible for low-mass stars to become carbon-rich because less carbon dredge-up is needed to dominate the thinning envelope.

\section{b) Visual and Infrared Carbon Stars}

Since $C$ stars include both visual and infrared $C$ stars (see $\S$ II), it would be interesting to compare the relative numbers of the two classes of carbon stars predicted by the model to observations. Models of Chan and Kwok (1990) suggest that the SiC feature becomes detectable when the envelope optical depth at $11.3 \mu \mathrm{m}\left(\tau_{0}\right)$ exceeds $\sim 0.05$, corresponding to a mass-loss rate of $\sim 2 \times 10^{-7} M_{\odot} \mathrm{yr}^{-1}$. When the continuum colors are considered, the photosphere begins to be significantly reddened at $\tau_{0} \sim 0.5$. Allowing for overlaps between the two classes (which is in fact observed), we arbitrarily draw the dividing line between visual and infrared $C$ stars at $\dot{M}=1 \times 10^{-6} M_{\odot}$ $\mathrm{yr}^{-1}$. Figure 8 shows the ratio of infrared to visual $\mathrm{C}$ stars as a function of $\chi$. The mass-loss formula of $\mathrm{BH}$ predicts a much higher fraction of infrared $\mathrm{C}$ stars, again because of the sudden increase in mass-loss rate near the end. If we ignore $\mathrm{BH}$ as totally unrealistic, the other formulae predict ratios in the range of $0.2-1$. The observed ratio is close to the low end of the theoretical values (or similar to those predicted by eq. [11]). Taking into account the fact that the infrared surveys are probably not as complete as visible surveys, the predicted ratio is consistent with observations.

In Figures 9 and 10, we have plotted the luminosity function for visual and infrared $C$ stars separately for the $\chi=0.06$ case. In the case of VK, the luminosity function of visual $\mathrm{C}$ stars is narrower and peaks at a lower luminosity than infrared $C$ stars. The two functions are similar for the mass-loss formula of equation (11).

\section{c) Luminosity Function of $M$ Stars}

Figure 6 shows that the luminosity function of $M$ stars peaks at $M_{\text {bol }} \sim-4.3$ and has a long tail extending to $M_{\text {bol }} \sim-7$. This is in excellent agreement with the observed luminosity function of $\mathbf{M}$ stars in external galaxies (Richer and Crabtree 1985; Pritchet et al. 1987). The difference in shape of the $M$ and C star luminosity function is due to the fact that high-mass $\left[M_{*}(0)>3 M_{\odot}\right]$ stars remain oxygen-rich for most of the AGB lifetime and $\mathrm{C}$ stars descend primarily from a lower mass $\left(<3 M_{\odot}\right)$ population.

Oxygen-rich stars on the AGB not only have the advantage of being more numerous, but their circumstellar spectra also contain more information than their carbon-rich counterparts because the $9.7 \mu \mathrm{m}$ silicate feature has been observed in 


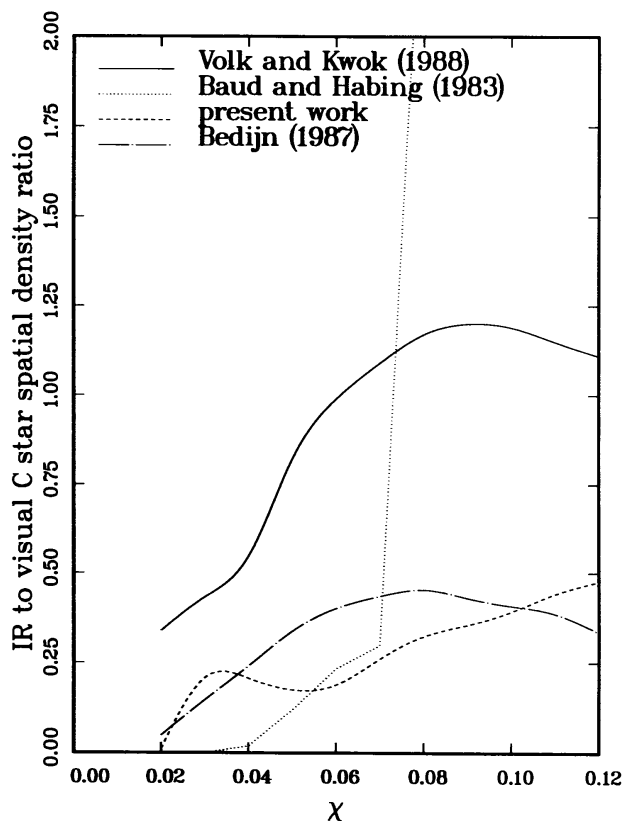

Fig. 8.-Predicted ratio of infrared to visual carbon stars as a function of the dredge-up parameter $\chi$ strengths from strong emission to strong absorption, corresponding to an optical depth range of over three orders of magnitude. Silicate absorption features have been detected by IRAS LRS in hundreds of stars (Volk and Kwok 1987), more can be detected by ground-based observations (Werner et al. 1980; Kwok, Hrivnak, and Boreiko 1987). The self-absorption of the silicate feature implies very high optical depths (from $\tau=5$ to 50 at $10 \mu \mathrm{m}$ ) in the circumstellar envelope, suggesting very high mass-loss rates. At these rates, the hydrogen envelope will be quickly depleted, implying the silicate absorption objects are close to the end of AGB evolution. Comparison of the Galactic distribution of silicate emission and absorption objects suggests that the latter probably originate from a higher initial mass population (Volk and Kwok 1987; Likkel 1989). If this is the case, then they probably represent the AGB stars in the luminosity gap between the upper limit of visual $C$ stars $\left(M_{\mathrm{bol}}=-6\right)$ and the AGB limit $\left(M_{\mathrm{bol}}=-7.1\right)$.

The fact that the $\mathrm{SiC}$ feature is not seen in absorption is also significant. Radiative transfer models suggest that the $\mathrm{SiC}$ will go into self-absorption at $\tau(11.3 \mu \mathrm{m})$ of $\sim 5$, or $\dot{M} \sim 2 \times 10^{-5}$ $M_{\odot} \mathrm{yr}^{-1}$. The absence of infrared C stars at very high massloss rates is consistent with the premise that $\mathrm{C}$ stars primarily originate from low-mass stars.

Since the change of the silicate feature from emission to absorption is strictly a function of the optical depth, we can use
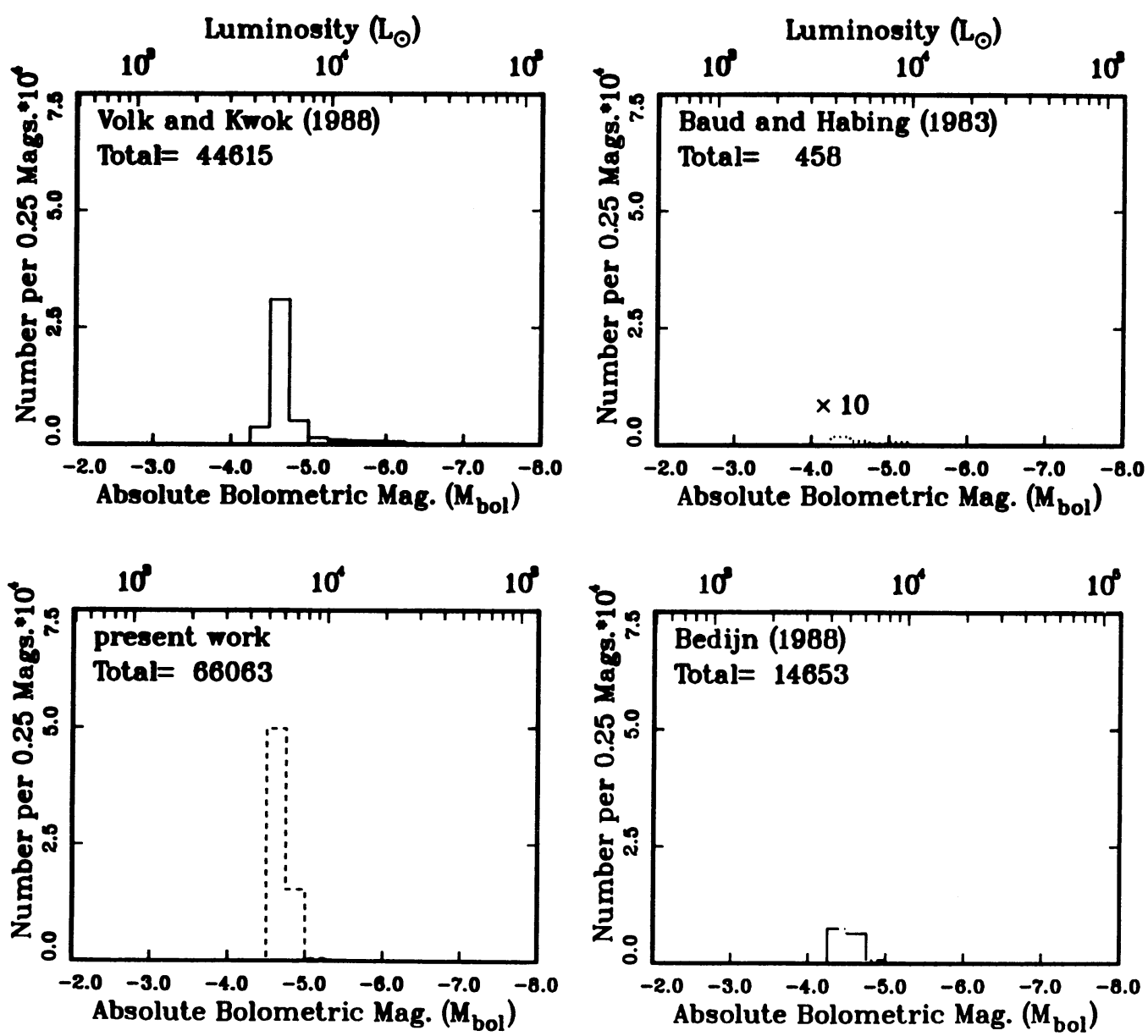

FIG. 9.-Model luminosity function of visual C stars 

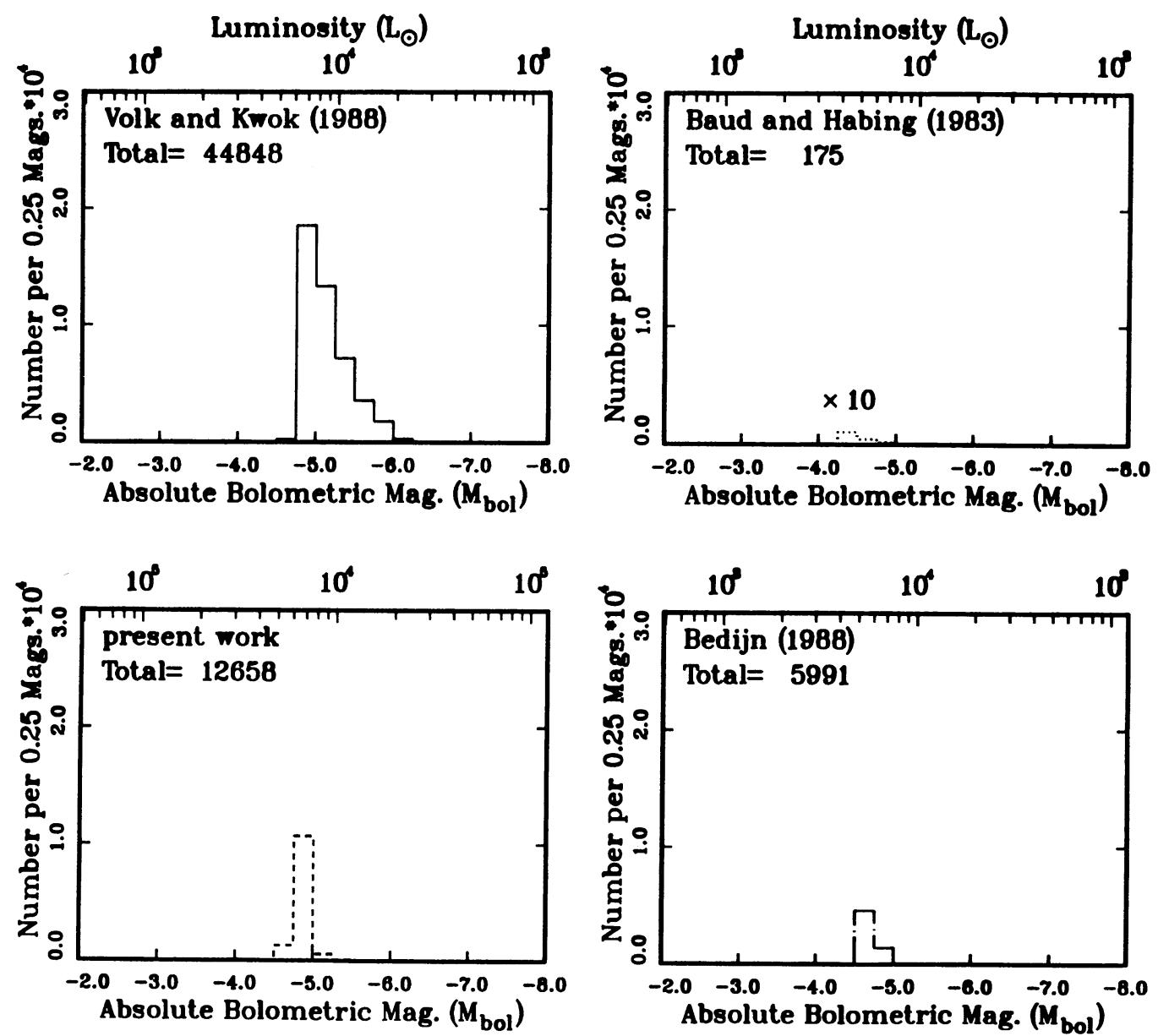

FIG. 10.-Model luminosity function of infrared $\mathrm{C}$ stars

the present model to predict the ratio of silicate emission to absorption objects. The radiative transfer models of Volk and Kwok (1988) suggest the change occurs at $\tau(10 \mu \mathrm{m}) \sim 4$, or approximately $\dot{M} \sim 8 \times 10^{-6} M_{\odot} \mathrm{yr}^{-1}$. Figure 11 shows the ratio of silicate absorption to emission objects as a function of $\chi$. The present mass-loss formula (eq. [11]) predicts a ratio of $2 \%-3 \%$. For comparison, the IRAS LRS Atlas contains $\sim 175$ and $>2000$ silicate absorption (class 30 , excluding $\mathrm{H}$ II regions) and emission objects (class 20, including some in class 10), respectively. The agreement between model and observations is reasonable if one considers the fact that the silicate absorption objects have higher luminosities and can be seen to a larger distance as is evident from their sky distributions.

\section{d) Dredge-up Models}

Previous theoretical work on the details of the dredge-up process has suggested the possible $\chi$ dependence on core mass. For example, in the models of Renzini and Voli (1981), $\chi$ changes monotonically from 0 at $M_{c}<0.6$ to 0.12 at $M_{c}>$ 1.26. We have tested the dredge-up formulation of Renzini and Voli (1981) with the mass-loss formulae used in this paper and found that far fewer carbon stars at low luminosities are produced. If there is indeed a low-mass cutoff for C stars, it can be produced with such $\chi$ dependence on core mass.

The present calculations suggest that carbon stars can be

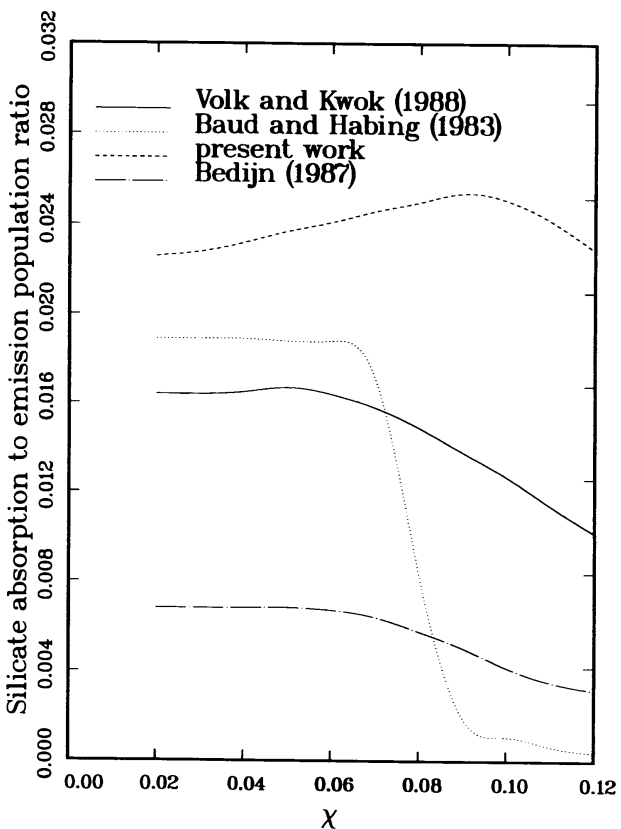

FIG. 11.--Predicted ratio of silicate absorption to emission objects as a function of the dredge-up parameter $\chi$. 
produced at $\chi$ between 0.04 and 0.08 if mass loss is taken into account. These values are consistent with the dredge-up parameters calculated by Lattanzio $(1989 a){ }^{3}$ although the model values of Lattanzio are a function of the thermal pulse number and are not a constant as assumed here. The effect of increasing the value of $\chi$ is that there will be more $\mathrm{C}$ stars at low luminosity, and the low-mass cutoff for the formation of $\mathrm{C}$ stars may disappear altogether. If the magnitude of mass loss is smaller or mass loss develops later than assumed here, then the value of $\chi$ has to be larger to produce similar numbers of $\mathrm{C}$ stars.

\section{e) Evolution from $M$ to $C$ Stars}

The spectral sequence of $M$ to $S$ to $C$ represents the changing photospheric composition of AGB stars and cannot be regarded as an evolutionary sequence for all AGB stars. The spectral sequence from M0 to M10 (and beyond) is both a mass sequence and an evolutionary sequence. $M$ stars branch to become $\mathrm{C}$ stars ${ }^{4}$ at different spectral types depending on the initial mass. For example, a low-mass star may evolve to a $\mathrm{C}$ star at spectral type of M6, whereas a higher mass star will change at spectral type beyond M10. A schematic Hertzsprung-Russell diagram is shown in Figure 12. The temperatures are calculated from equation (7) and the corresponding spectral types are taken from Querci (1986).

Figure 12 can be considered as only a schematic portrayal of the evolution on the AGB because the temperature formula (eq. [7]) is only approximate. Better representations of the AGB evolution are the Wood and Cahn (1977) diagrams (see Fig. 3) and color-color diagrams (Bedijn 1987; Volk and Kwok 1988; Chan and Kwok 1988, 1990). The color-color diagram is particularly useful because AGB stars undergo large changes in infrared colors during their evolution but only slight changes in photospheric temperature which are difficult to determine. A complete representation of evolutionary tracks on the color-color diagram will require a radiative transfer calculation for each step of the AGB, taking into account the changes and interruption in the mass-loss process.

\section{f) The End Point of Asymptotic Giant Branch}

The end point of AGB can be defined as when the envelope mass has been depleted to $<10^{-3} M_{\odot}$ (depending on core mass; Schönberner 1983) by mass loss and the effective temperature of the star begins to increase rapidly. Large-scale mass loss will cease and the star will evolve through the protoplanetary nebula stage to a planetary nebula in $\sim 1000 \mathrm{yr}$ (Volk and Kwok 1989). Since the planetary nebula is made up of material ejected by the star during the last $10^{4} \mathrm{yr}$, whether the planetary nebula will be oxygen- or carbon-rich will depend on the chemical composition of the envelope when $M_{e}$ falls below $0.1 M_{\odot}$.

Figure 13 shows the fraction of AGB stars that are carbon rich at the end of AGB. With $\chi=0.07$ and the mass-loss formula in equation (11), approximately half of all planetary nebulae are expected to be carbon-rich. This is consistent with the observed fraction of $60 \%$ as determined by Zuckerman and Aller (1986).

\section{CONCLUSIONS}

Recent analysis of IRAS data has revealed that there are three different kinds of AGB stars based on their circumstellar

\footnotetext{
${ }^{3} \lambda$ in Lattanzio $(1989 a)$ is approximately 4 times the value of $\chi$.

${ }^{4}$ More specifically, $\mathrm{N}$ stars in common spectroscopic nomenclature.
}

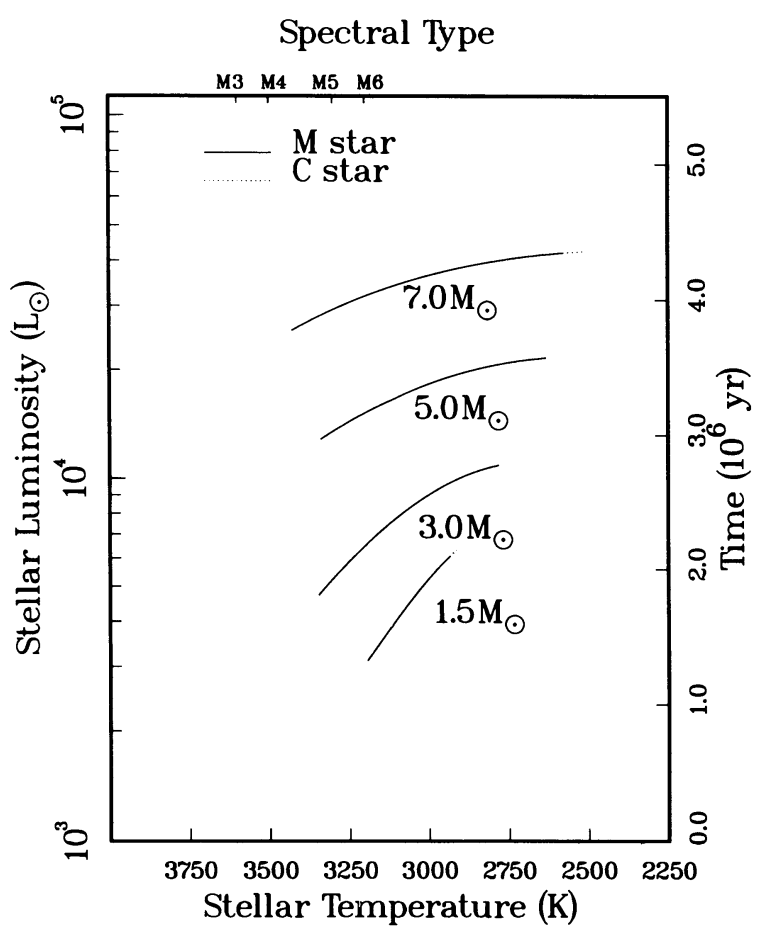

FIG. 12.-A schematic H-R diagram for AGB evolution for stars with initial masses $1.25,3,5$, and $7 M_{\odot}$. The mass-loss formula in eq. (11) is used. The solid and dashed lines represent the oxygen- and carbon-rich phases, respectively.

spectra: $\mathrm{M}$ stars, visual $\mathrm{C}$ stars, and infrared $\mathrm{C}$ stars. There is also the additional class of $S$ stars whose origin is uncertain. The spectra of these three groups suggest that $\mathbf{M}$ stars and infrared $\mathrm{C}$ stars are losing mass but visual $\mathrm{C}$ stars are in an evolutionary phase between two mass-losing phases. These discoveries have led to the development of a new integrated

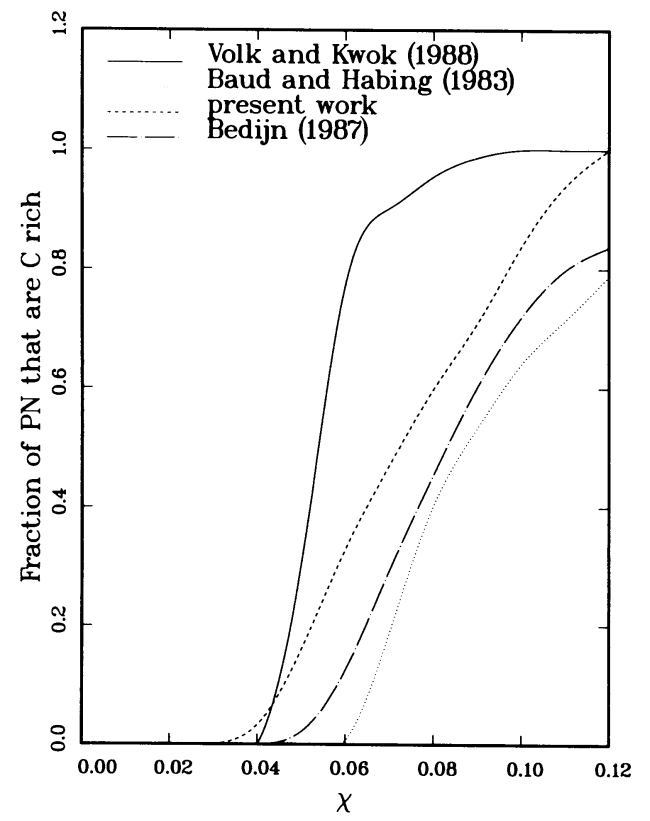

FIG. 13.-Predicted fraction of stars that are carbon-rich at the end of AGB. 
scenario of AGB evolution. We suggest that stars with high (3-8 $M_{\odot}$ ) initial masses will stay oxygen-rich most of their lifetime on the AGB whereas stars with lower initial masses will go through the carbon-rich phase, first as visual $\mathrm{C}$ stars and later as infrared $C$ stars. Using a simple dredge-up formula, we are able to reproduce the observed luminosity functions of $\mathrm{M}$ and $\mathrm{C}$ stars.

In order to form $\mathrm{C}$ stars at low luminosities (and core masses), we find that the mass-loss rate must be significant (in comparison to the nuclear burning rate) during the entire AGB. The "superwind" mass-loss formulae, which remove most of the envelope mass near the end of the AGB, produce far fewer $\mathrm{C}$ stars than are observed.
The calculations presented here represent the first attempt at an integrated AGB evolution model taking into account all major physical processes. While certain aspects of the details of the model need further refinement, the model is successful in reproducing the photospheric and circumstellar properties of AGB stars. We confirm the importance of mass loss as a process that is significant not only near the end but throughout the entire AGB.

This work is supported by a grant to S. K. from the Natural Sciences and Engineering Research Council of Canada.
Baud, B., and Habing, H. J. 1983, Astr. Ap., 127, 73 (BH). 1987, Astr. Ap., 186, 136 (B87)

1988, Astr. Ap., 205, 105

Blanco, B. M., McCarthy, M. F., and Blanco, V. M. 1978, Nature, 271, 638. 1980, Ap. J., 242,938.

Boothroyd, A. I., and Sackmann, I.-J. 1988a, Ap. J., 328, 641 $1988 b, A p . J ., 328,653$. $1988 c, A p . J, 328,671$

Castellani, V., Chieffi, A., Pulone, L., and Tornambè, A. 1985, Ap. J., 296, 204.

Chan, S. J., and Kwok, S. 1988, Ap. J., 334, 362. 1990, Astr. Ap., in press.

Chiosi, C., Bertelli, G., and Bressan, A. 1987, in Late Stages of Stellar Evolution ed. S. Kwok and S. R. Pottash (Dordrecht: Reidel), p. 213.

Cohen, J. G., Forgel, F. J., Persson, S. E., and Elias, J. H. 1981, Ap. J., 249, 481

Hollowell, D. E. 1987, in Late Stages of Stellar Evolution, ed. S. Kwok and S. R. Pottasch (Dordrecht: Reidel), p. 239.

Iben, I. 1981, Ap. J., 246, 278. $1982, A p . J ., 208,165$

. 1988, in Progress and Opportunities in Southern Hemisphere Optical Astronomy, ed. V. M. Blanco and M. M. Phillips (A.S.P. Conf. Ser., 1), p. 220. Iben, I., and Renzini, A. 1982, Ap. J. (Letters), 259, L79. 1983, Ann. Rev. Astr. Ap., 21, 271.

Iben, I., and Rood, R. T. 1970, Ap. J., 161, 587.

Kwok, S., Hrivnak, B. J., and Boreiko, R. T. 1987, Ap. J., 321, 975.

Kwok, S., Volk, K. M., and Chan, S. J. 1989, in Evolution of Peculiar Red Giant Stars, ed. H. Johnson and B. Zuckerman (Cambridge: Cambridge University Press), p. 284.

Lattanzio, J. C. $1989 a$, Ap. J. (Letters), 344, L27. $1989 b$, in Evolution of Peculiar Red Giant Stars, ed. H. Johnson and B. Zuckerman (Cambridge: Cambridge University Press), p. 161

Likkel, L. 1989, Ap. J., 344, 350.

Little-Marenin, I. R. 1986, Ap. J. (Letters), 307, L15.

MacConnell, D. J. 1988, A.J., 96, 354.

Merrill, K. M. 1977, in IAU Colloquium 42; The Interaction of Variable Stars with Their Environment, ed. R. Kippenhahn, J. Rahe, and W. Strohmeier (Veröff. Remeis-Sterw. Bamberg, Vol. 11, No. 121), p. 446.

\section{REFERENCES}

Miller, G. E., and Scalo, J. M. 1979, Ap. J. Suppl., 41, 513.

Paczyński, B. 1971, Acta Astr., 31, 417.

. 1977, Ap.J., 214,812.

Pritchet, C. J., Richer, H. B., Schade, D., Crabtree, D., and Yee, H. K. C. 1987, Ap. J., 323, 79.

Querci, F. R. 1986, in The M-Type Stars, ed. H. R. Johnson and F. R. Querci (NASA SP-492), p. 1

Renzini, A., and Voli, M. 1981, Astr. Ap., 94, 175.

Richer, H. B. $1981, A p . J ., 243,744$.

Richer, H. B., and Crabtree, D. R. 1985, Ap. J.(Letters), 298, L13.

Salpeter, E. E. 1955, Ap. J., 121, 161.

Schönberner, D. 1983, Ap. J., 272, 708.

Stephenson, C. B. 1973, A General Catalogue of Cool Carbon Stars, Pub. Warner and Swasey Obs., Vol. 1, No. 4

Thronson, H. A., Latter, W. B., Black J. H., Bally, J., and Hacking, P. 1987, Ap. $J ., 322,770$.

Volk, K., and Kwok, S. 1987, Ap. J., 315, 654

-. 1988, Ap. J., 331, 435(VK).

- 1989, Ap.J., 342, 345

Walker, H. J., and Cohen, M. J. 1988, A.J., 95, 1801

Weidemann, V., and Koester, D. 1983, Astr. Ap., 121, 77

Willems, F. J., and de Jong, T. 1988, Astr. Ap., 196, 171.

Werner, M. W., Beckwith, S., Gatley, I., Sellgren, K., Berriman, G., and Whiting, D. L. 1980, Ap. J., 239, 540 .

Wood, P. R. 1981, in Physical Processes in Red Giants, ed. I. Iben, Jr., and A. Renzini (Dordrecht: Reidel), p. 135.

. 1987, in Late Stages of Stellar Evolution, ed. S. Kwok and S. R. Pottasch (Dordrecht: Reidel), p. 197.

Wood, P. R., Bessell, M. S., and Fox, M. W. 1983, Ap. J., 272, 99.

Wood, P. R., and Cahn, J. H. 1977, Ap. J., 211, 499.

Zuckerman, B., and Aller, L. H. 1986, Ap. J., 301, 772.

G. BRYAN and K. VolK: Department of Physics and Astronomy, University of Calgary, Calgary, Alberta, Canada T2N 1N4

S. KwoK : Joint Institute for Laboratory Astrophysics, Campus Box 440, University of Colorado, Boulder, CO 80309 